\title{
Valence bonds for different people
}

\author{
Explanations of high-temperature superconductivity seem to be settling about the concept of the \\ valence bond, which appears to mean different things to different people.
}

THE rapprochement between chemistry and physics stimulated by the search for an explanation of high-temperature $(90 \mathrm{~K})$ superconductivity evidently goes deeper than might have been expected. So much is plain from the material appearing in physics journals, but there is a long way to go before the rapprochement will be complete. For the time being, there seems to be a string of semantic issues to be resolved, people's understanding of what is meant by a valence bond, for example, a notion that seems all the more important now that P.W.Anderson seems to be winning people round to his view that resonating patterns of valence bonds are at the root of the new phenomena.

Part of the trouble is that chemists and physicists use different language to refer to the same concept and part of it is that that they also mean slightly different things when they say "valence bond". Some of the confusion stems from the way in which people are taught their crafts, but there is little doubt that what matters most to a chemist about a valence bond is that it should be strong enough to hold a molecule, or some part of it, together.

Physicists, however, talk as if all that matters about a valence bond is that it is a device for satisfying Pauli's exclusion principle - that two electrons cannot be in the same state at the same time unless their spins are antiparallel. This seems to be the reason why the words physicists use for the discussion of valence-bond patterns in solids are often simple adaptations of those used for the discussion of magnetism (antiferromagnetism as well as ferromagnetism). Each lattice point carries a spin, the energy of the interactions between a pair of neighbours is proportional to the product of the values of the two spins (with a constant of proportionality which is negative in the case of a ferromagnet). So does it not make sense to think of a valence-bonded solid structure as the ground state of a spin-loaded lattice in which the constant of proportionality is positive, favouring neighbouring antiparallel spins?

In many ways, the notation is simplifying. It has a great success in discussions of polyacetylene, for example, where if the backbone of the molecule is thought of as held together by inviolable sigma bonds between adjacent carbon atoms, the question is merely how to dispose of the spare pair of electrons on each carbon atom. But there are circumstances when the rep- resentation of valence bonds as neighbourneighbour spin couplings can be misleading. Although it has now emerged, under the impetus of the hunt for an explanation of superconductivity, that $\mathrm{La}_{2} \mathrm{CuO}_{4}$ is indeed an antiferromagnetic material, it is also the case that the nearest-neighbour interactions responsible for magnetic phenomena are not nearly as strong as those responsible for valence bonding.

More seriously, although the simple spin representation of one-dimensional polymers with spare electrons has neatly pointed to the importance of soliton solutions (say a break in a normally regular alternation of bond types), an unusual Chinese Japanese collaboration (Changqin Wo and Xin Sun from Fudan University, Shanghai, and Keiichiro Nasu from the Molecular Science Institute at Okazaki) has now shown how great are the complications of attempting to allow for electron-electron interactions even in simple one-dimensional polymers (Phys. Rev. Lett. 59, 831; 1987).

It is remarkable that the group is able to say anything at all about the dependence of the alternation of bond lengths on the parameters defining the problem, the strength of the interaction between electrons and the lattice, or the range of their interaction with each other (which depends on the polarizability of the backbone bonds, among other things). Yet these problems may ultimately have to be faced by those who would use valencebond models to account for high-temperature superconductivity.

With all that said, the formal spincoupling representations of valence bond lattices do appear to be making some headway, most remarkably in an article by R.H. Parmenter of the University of Arizona (Phys. Rev. Lett. 59, 923; 1987), who constructs a hamiltonian for a crystal lattice (or even two interpenetrating lattices) accommodating spin-spin interactions between nearest neighbours and between next-nearest neighbours by means of an intermediate atom (shades of the $\mathrm{Cu}-\mathrm{O}-\mathrm{Cu}$ chains in the yttrium copper oxide structures).

Parmenter concludes that, with a suitable choice of parameters, a lattice can be at once antiferromagnetic (demonstrated by long-range order) and superconducting, which contradicts one of P.W.Anderson's more recent conclusions (with $G$. Baskaran, Z. Zou and T. Hsu in Phys. Rev. Lett. 58, 2790; 1987) that the electron holes created by the movement of valence bonds are inimical to antiferromagnetism. But if there should be exactly one electron for each lattice site, the antiferromagnetism will cause the electron band to be filled and superconductivity will not be possible.

The argument by Anderson and his colleagues is interesting because it makes more explicit than in previous papers the presumed link between the phase transition in $\mathrm{La}_{2} \mathrm{CuO}_{4}$ from a tetragonal to an orthorhombic crystal structure (which takes place at $550 \mathrm{~K}$ for the undoped material, but at lower temperatures when barium is substituted for lanthanum) and the occurrence of superconductivity. The distortion of the tetragonal crystal structure, this view says, signals the arrival of a phase in which resonating valence bonds are dominant, with quite substantial fractions of an electron charge transferred to the intraplane oxygens (already supposed to be doubly negative). But in those circumstances, the square symmetry in the plane will be broken, making each a parallelepiped.

On this view, say Anderson and his colleagues, exactly the parameters that control the phase transition in crystal structure also control the transition to superconductivity at lower temperatures. In particular, they say, these parameters determine that the superconducting transition temperature should increase with the proportion of barium doping while the crystal transition temperature falls.

What will the chemists make of all this? They will probably acknowledge that the representation of interacting atoms by quantum spin operators is unavoidable, given that there is an infinite lattice to handle. The physical difference between the application of valence-bond methods to simple molecules such as that of hydrogen and to lattice structures is that, for the molecule, it is necessary to take account of bizarre configurations in which both electrons surround one or other of the nuclei, leaving the other bare. In a crystal, it is much easier to suppose that there should be a single extra charge, or a few of them, on some of the interacting atoms, which is another way of saying that there will be a plethora of modestly excited states that common sense suggests should be counted, which is what the formalism of spin operators does. The snag is that it will take some time before everybody tumbles to this.

John Maddox 